\title{
Surgical Management of Unicentric Castleman's Disease in the Abdomen
}

\author{
Min-Sang Kim, Jae-Kyun Ju, Young Kim ${ }^{1}$ \\ Departments of Surgery and ${ }^{1}$ Pathology, Chonnam National University Medical School, Gwangju, Korea
}

Castleman's disease (CD) is a rare lymphoproliferative disorder that can involve single or multiple lymph nodes in the body. Especially, the localized form of $\mathrm{CD}$ is known to be well-controlled by using a surgical resection. On occasion, the surgeon may confront an abdominal and retroperitoneal mass of unknown origin. Thus, we present this case in which we treated a 16-year-old female patient for $\mathrm{CD}$ and investigated how to evaluate and manage the situation from the standpoint of CD. Also, we give a review of the pathology, clinical manifestation, diagnosis, and treatment of CD.

Keywords: Giant lymph node hyperplasia

\section{INTRODUCTION}

Castleman's disease (CD) is a rare lymphoproliferative disorder and was first described in 1956 by Castleman et al. [1] as a benign, localized enlargement of hyperplastic lymph nodes. The pathogenesis of that disease is known to be related to the human herpes virus 8 (HHV8) [2]. Generally, the disease is divided into two groups depending on its histologic and clinical features. In 1972, Keller et al. [3] subclassified CD into a hyaline-vascular (HV) type and a plasma-cell (PC) type based on their histologic features. Some patients have a mixed form. Clinically, $\mathrm{CD}$ can be divided into a localized form, which refers to a unicentric (UC) disease, and a generalized form, which refers to a multicentric (MC) disease.

UC CD is predominantly developed in the mediastinum or the lung hilum. However, the abdomen and the retroperitoneum may also be involved [4]. When UC-type CD is managed by using a

Received: July 24, 2013 • Accepted: December 24, 2013

Correspondence to: Jae-Kyun Ju, M.D.

Department of Surgery, Chonnam National University Hospital, Chonnam National University Medical School, 42 Jebong-ro, Dong-gu, Gwangju 501-757, Korea

Tel: +82-62-220-6456, Fax: +82-62-227-1635

E-mail: jkju@jnu.ac.kr

(C) 2014 The Korean Society of Coloproctology

This is an open-access article distributed under the terms of the Creative Commons Attribution NonCommercial License (http://creativecommons.org/licenses/by-nc/3.0) which permits unrestricted noncommercial use, distribution, and reproduction in any medium, provided the original work is properly cited. successful surgical resection, the outcome is usually more favorable than it is for MC-type CD [5]. Thus, differentiating between the types of $\mathrm{CD}$ is important.

We introduce the case of a 16-year-old female patient who had UC CD, and based on that experience, we discuss how to evaluate and manage UC CD. Also, we review the pathology, clinical manifestation, diagnosis, and treatment of $\mathrm{CD}$.

\section{CASE REPORT}

A 16-year-old female patient with neither a past medical history nor a family history of $\mathrm{CD}$ presented at another medical facility with right lower quadrant pain and vomiting for 3 days. She was initially evaluated by using ultrasonography and computed tomography $(\mathrm{CT})$ of the abdomen. Ultrasonography showed a retroperitoneal $4.7-\mathrm{cm}$ mass near the vena cava on the umbilicus level. The mass was characterized by hypervascularity on the enhanced abdominal CT.

Based on the above imaging findings, the patient visited to our hospital as an outpatient. Physical examination revealed a right abdominal mass on deep palpation. No other lymph node enlargements were present on physical examination. Chest and abdominal $\mathrm{x}$-ray films were considered normal. The blood-analysis results were normal. On the viral screening test, the human immunodeficiency virus (HIV) screening test was negative. The magnetic resonance imaging of the patient's abdomen for the diagnosis revealed a $5.0 \mathrm{~cm}^{3} \times 4.0 \mathrm{~cm}^{3} \times 2.8 \mathrm{~cm}^{3}$ well-defined right 

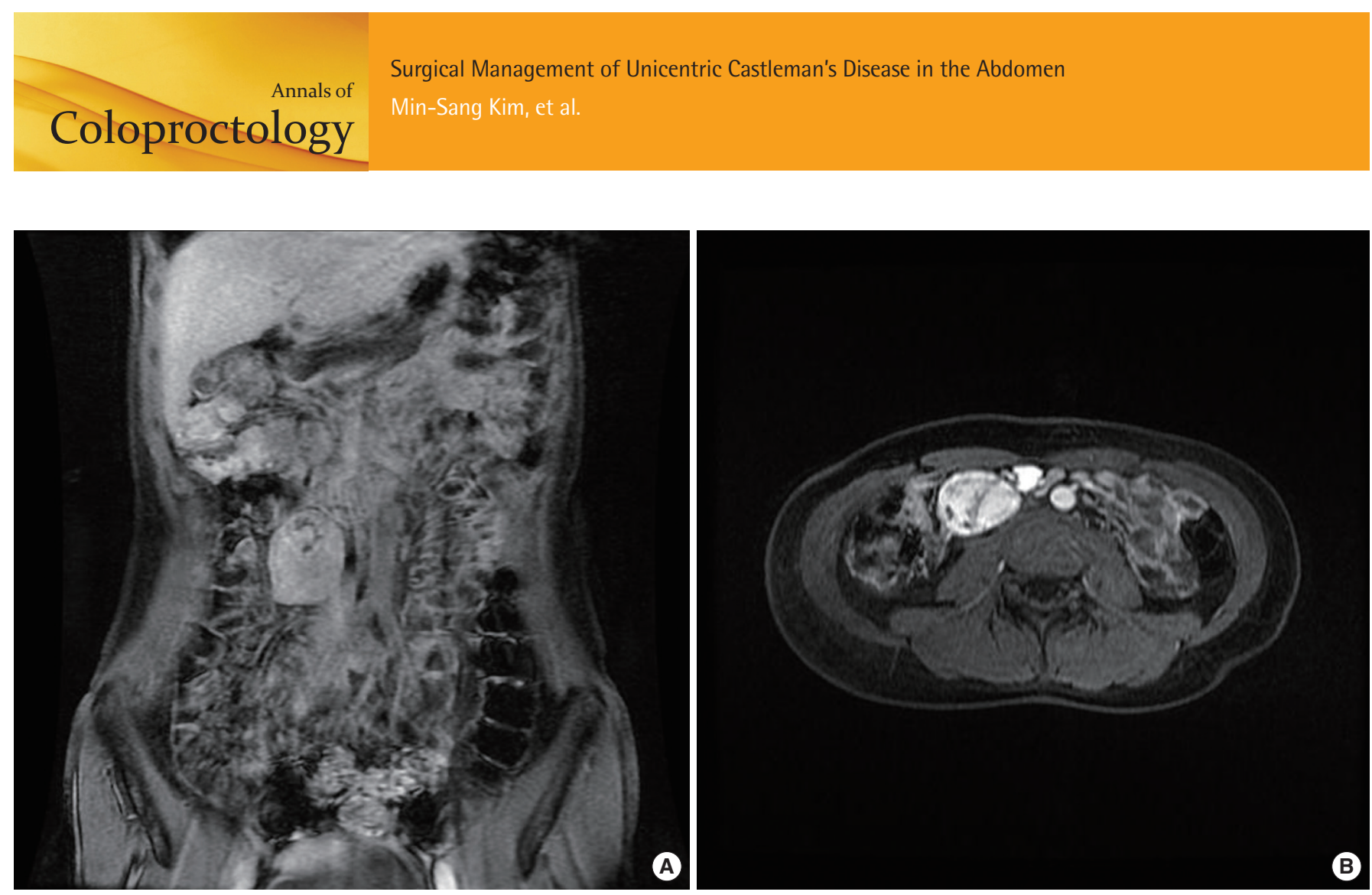

Fig. 1. T1-weighted magnetic resonance images showing a well-defined enhanced mass, about $5 \mathrm{~cm} \times 4 \mathrm{~cm} \times 2.8 \mathrm{~cm}$, with hypervascular and heterogenic characteristics in the right retroperitoneum, suggesting rule out Castleman's disease. (A) Coronal view, (B) Transverse view.

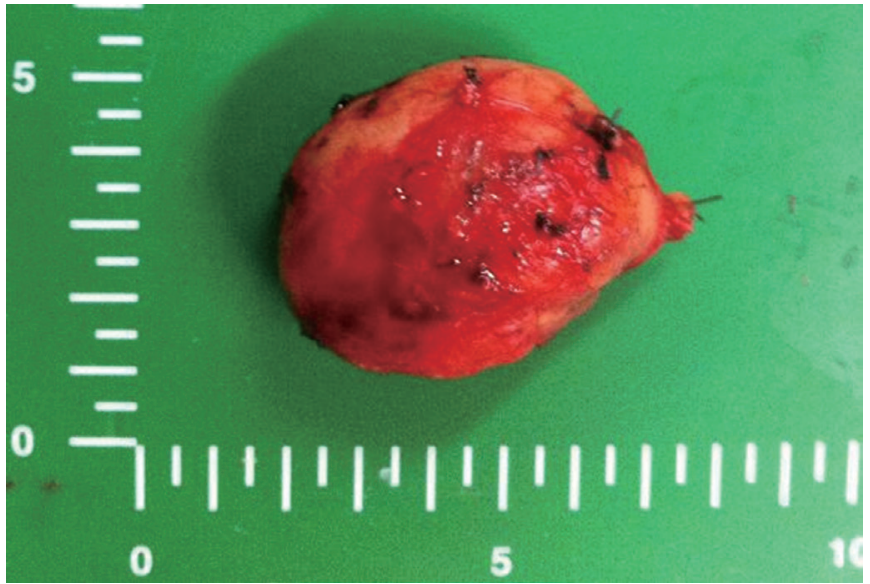

Fig. 2. Gross finding.

retroperitoneal mass abutting the infrarenal vena cava. The mass showed a heterogeneous pattern and bright enhancement following an intravenous contrast injection. Surgery was planned with a preoperative diagnosis of a retroperitoneal tumor (Fig. 1).

During the laparotomy, a $5.5 \mathrm{~cm}^{3} \times 3.5 \mathrm{~cm}^{3} \times 3.0 \mathrm{~cm}^{3}$ round mass was found in the retroperitoneal space, just inferior to the right kidney. The mass, which was not invading other organs, was completely excised (Fig. 2). After surgery, the patient well recovered well without any other complications. She has been free of disease for more than 18 months since the resection.

The histologic diagnosis of the surgically-resected specimen was a hyaline vascular (HV) type of CD. The microscopic examination of this mass showed follicles with expanded mantle zones containing germinal centers and prominent interfollicular vascular proliferation. Residual germinal centers penetrated by hyalinized vessels and small lymphocytes palisaded around the germinal centers, imparting an "onion-skin" appearance. CD21 immunohistochemical staining showed a tight follicular dendritic meshwork within an atrophic germinal center extending in a loosely-arranged pattern into the mantle zone (Fig. 3).

\section{DISCUSSION}

The clinical presentations of CD are not specific for CD and are different between the UC and the MC types of CD. The UC type of $\mathrm{CD}$ is associated with mass-effect symptoms related to the compression of adjacent organs. Patients complain of postprandial discomfort, vomiting, weight loss, urinary retention and abdominal or lumbar pain [4]. The MC type of CD is associated with systemic disturbances, such as anemia, increased erythrocyte sedimentation rate, polyclonal hypergammaglobulinemia, hypoalbuminemia and thrombocytopenia, which can be associated with symptoms like asthenia, fever, weight loss, generalized lymphadenopathy, hepatomegaly, splenomegaly, peripheral edema, pleural effusion, impaired renal function and sometimes polyneuropathy [4]. As mentioned earlier, the clinical presentations of CD are various, so diagnosing CD by using clinical symptoms is difficult. Clinicians should proceed with other evaluations based on the symptoms. 

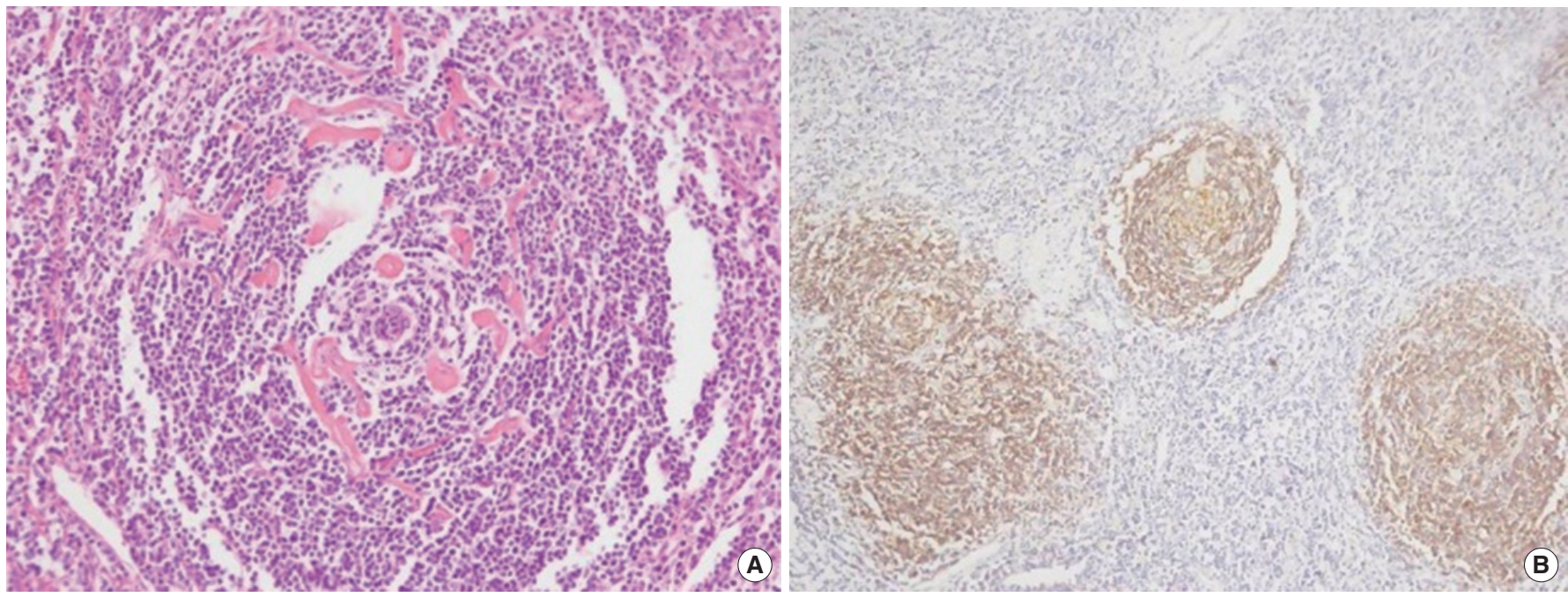

Fig. 3. (A) "Onion-skin" appearance (H\&E staining, $\times 400)$. (B) CD21 immunohistochemical staining $(\times 100)$.

Generally, clinicians perform imaging investigations such as enhanced CT at the site of the patient's symptoms. However, because of the heterogenecity and the hypervascularity of the retroperitoneal mass of $\mathrm{CD}, \mathrm{CD}$ is usually not considered as the first diagnosis [6]. Also, $80 \%$ of retroperitoneal tumors are malignant [7]. A lymphoma, soft-tissue sarcoma or vascular tumor are possible diagnoses. Urologic tumors such as seminomas, prostatic cancer, and teratomas tend to give rise to metastatic disease in the retroperitoneum in the form of an adenopathy, generally multiple adenopathies [8]. Because of the lack of specific investigations of CD, clinicians facing a retroperitoneal tumor should consider $\mathrm{CD}$ as a possible diagnosis. Although surgery is planned, macroscopically differentiating $\mathrm{CD}$ from other malignant tumors is nearly impossible. Because CD lesions harbor dense fibrous adherences to adjacent organ and because hypervacularization is typically seen in malignant pathology [9], open frozen biopsy during operation may be helpful. It may allow an extensive resection, especially a resection of a nearby organ not invaded by this benign cancer, to be avoided [4].

The pathogenesis of CD is to be related to HHV8 as HHV8 has been found in lymphoid cells in the systemic form of CD. Chronic infection by HHV8 stimulates secretion of interleukin (IL) 6 , which, in turn, induces a hyperplastic reaction of the lymphoid system [10]. Elevated serum levels of IL-6 and demonstration of circulating HHV8 particles via polymerase chain reactions can lead to the diagnosis, but are not commonly used in clinical practice [11]. Also, HIV-associated CD is almost always MC and strongly correlates to HHV8 [12]. In our case, we performed an HIV screening test, and the result was negative. If the MC type of $\mathrm{CD}$ is clinically suspected, the existence of HIV and HHV8 should be evaluated.

If $\mathrm{CD}$ is a possible diagnosis, then determining its type (UC or $\mathrm{MC})$ is the most important thing. In a recent study that under- took a systemic review of 404 published cases of CD, UC type was superior to MC type in clinical outcomes and long-term followup results. In MC disease, the role of surgery is limited to gaining tissue by biopsy and to debulking the tumor to address specific problems such as bowel obstruction, vascular or airway compromise, and massive organomegaly [5]. However, in UC disease, the en bloc resection of the tumor is the best treatment option. If the resection is successful, the clinical outcome will be very satisfactory, and no further treatment will be needed. Failure to resect the primary involved lymph node is the only significant predictor for a fatal outcome, and an incomplete resection has a significantly worse outcome than a complete excision [5]. Therefore, clinicians should investigate the type of $\mathrm{CD}$ and remove the tumor completely when the $\mathrm{CD}$ is the UC type.

$\mathrm{CD}$ is histopathologically subclassified into two types. The HV type is characterized by giant lymph follicles centered on a central vessel with marked hyalinization. The PC type contains many more polyclonal plasma cells with a less marked hyalinization and vascularization [3]. Approximately $90 \%$ of all localized forms are the HV type, but most of the systemic forms are the PC type [13]. Some studies suggest that the histopathologic type should primarily be used for classification purposes $[14,15]$. However, no differences in outcomes were noted when comparing the PC and the HV types in the patient groups with UC or MC disease [5].

In conclusion, when a solitary retroperitoneal and abdominal mass is present, $\mathrm{CD}$ may be a possible diagnosis. When the UC type of $\mathrm{CD}$ is suspected, the surgeon should remove the mass completely.

\section{CONFLICT OF INTEREST}

No potential conflict of interest relevant to this article was reported. 


\section{REFERENCES}

1. Castleman B, Iverson L, Menendez VP. Localized mediastinal lymphnode hyperplasia resembling thymoma. Cancer 1956;9:822-30.

2. Schulte KM, Talat N. Castleman's disease: a two compartment model of HHV8 infection. Nat Rev Clin Oncol 2010;7:533-43.

3. Keller AR, Hochholzer L, Castleman B. Hyaline-vascular and plasma-cell types of giant lymph node hyperplasia of the mediastinum and other locations. Cancer 1972;29:670-83.

4. Bucher P, Chassot G, Zufferey G, Ris F, Huber O, Morel P. Surgical management of abdominal and retroperitoneal Castleman's disease. World J Surg Oncol 2005;3:33.

5. Talat N, Belgaumkar AP, Schulte KM. Surgery in Castleman's disease: a systematic review of 404 published cases. Ann Surg 2012;255: 677-84.

6. Fields S, Bar-Ziv J, Portnoy O, Sasson T, Sherman Y, Libson E. Radiologic spectrum of localized Castleman's disease. Isr J Med Sci 1995;31:660-9.

7. Papanicolaou N, Yoder IC, Lee MJ. Primary retroperitoneal neoplasms: how close can we come in making the correct diagnosis. Urol Radiol 1992;14:221-8.

8. Lane RH, Stephens DH, Reiman HM. Primary retroperitoneal neoplasms: CT findings in 90 cases with clinical and pathologic correlation. AJR Am J Roentgenol 1989;152:83-9.

9. Seco JL, Velasco F, Manuel JS, Serrano SR, Tomas L, Velasco A. Retroperitoneal Castleman's disease. Surgery 1992;112:850-5.

10. Shahidi H, Myers JL, Kvale PA. Castleman's disease. Mayo Clin Proc 1995;70:969-77.

11. Hengge UR, Ruzicka T, Tyring SK, Stuschke M, Roggendorf M, Schwartz RA, et al. Update on Kaposi's sarcoma and other HHV8 associated diseases. Part 2: pathogenesis, Castleman's disease, and pleural effusion lymphoma. Lancet Infect Dis 2002;2:344-52.

12. Oksenhendler E, Duarte M, Soulier J, Cacoub P, Welker Y, Cadranel J, et al. Multicentric Castleman's disease in HIV infection: a clinical and pathological study of 20 patients. AIDS 1996;10:61-7.

13. Herrada J, Cabanillas F, Rice L, Manning J, Pugh W. The clinical behavior of localized and multicentric Castleman disease. Ann Intern Med 1998;128:657-62.

14. Cronin DM, Warnke RA. Castleman disease: an update on classification and the spectrum of associated lesions. Adv Anat Pathol 2009;16:236-46.

15. McClain KL, Natkunam Y, Swerdlow SH. Atypical cellular disorders. Hematology Am Soc Hematol Educ Program 2004:283-96. 